\title{
A Novel Filled Function Method for Nonlinear Equations
}

\author{
Liuyang Yuan ${ }^{1}$ and Qiuhua Tang ${ }^{2}$ \\ ${ }^{1}$ College of Sciences, Wuhan University of Science and Technology, Wuhan, Hubei 430081, China \\ ${ }^{2}$ Industrial Engineering Department, Wuhan University of Science and Technology, Wuhan, Hubei 430081, China
}

Correspondence should be addressed to Liuyang Yuan; yangly0601@126.com

Received 24 December 2013; Accepted 26 May 2014; Published 12 June 2014

Academic Editor: Nan-Jing Huang

Copyright (C) 2014 L. Yuan and Q. Tang. This is an open access article distributed under the Creative Commons Attribution License, which permits unrestricted use, distribution, and reproduction in any medium, provided the original work is properly cited.

\begin{abstract}
A novel filled function method is suggested for solving box-constrained systems of nonlinear equations. Firstly, the original problem is converted into an equivalent global optimization problem. Subsequently, a novel filled function with one parameter is proposed for solving the converted global optimization problem. Some properties of the filled function are studied and discussed. Finally, an algorithm based on the proposed novel filled function for solving systems of nonlinear equations is presented. The objective function value can be reduced by quarter in each iteration of our algorithm. The implementation of the algorithm on several test problems is reported with satisfactory numerical results.
\end{abstract}

\section{Introduction}

Systems of nonlinear equations arise in myriad applications, for example, in engineering, physics, mechanics, applied mathematics and sciences; see [1] for a more detailed description.

In this paper, we consider the following box-constrained systems of nonlinear equations (for short, (SNE)):

$$
F(x)=0, \quad x \in X,
$$

where the mapping $F: R^{n} \rightarrow R^{m}$ is continuous, $x \subset R^{n}$ is a box.

Generally, systems of nonlinear equations are very difficult to solve directly. The typical methods to solve (SNE) are optimization-based methods in which (SNE) is reformulated as an optimization problem. The most popular optimizationbased methods involve solving the following optimization problem (for short (OP)):

$$
\begin{aligned}
& \min \quad f(x)=\frac{1}{2} F(x)^{T} F(x) \\
& \text { s.t. } \quad x \in X
\end{aligned}
$$

to find solutions of (SNE). Note that the problem above is a box-constrained nonlinear least-squares problem. It is easy to see that the objective function satisfies $f(x) \geq 0$ and global optimal solutions of problem (OP) with the zero objective function value corresponding to solutions of (SNE).

Generally speaking, the traditional optimization-based methods for solving (SNE) are often stuck at a stationary point or a local minimizer of the corresponding optimization problem, which is not necessarily a solution of the original system. Lately, great efforts have been made to overcome the difficulty caused by nonglobal minimizers. In Particular, some switching techniques [2-6] have been developed to escape from a stationary point or a local minimizer which is not a solution of (SNE).

Kanzow [3] incorporated two well-known global optimization algorithms, namely, a tunneling [7] and a filled function method [8], into a standard nonsmooth Newton-type method for solving a nonsmooth system of equations which is a reformulation of the mixed complementarity problem. Wu et al. [9] and Lin et al. [10, 11] also gave some filled function methods to solve a nonlinear system with box constraints. Wang et al. [12] gave a filled function method to solve an unconstrained nonlinear system. In this paper, we will propose another kind of filled function method to solve boxconstrained systems of nonlinear equations. Unlike [3], we do not use any Newton-type methods to solve (SNE), and, also unlike $[9,10]$, a better initial point of the primal optimization 
problem sometimes can not be obtained by minimizing the constructed filled function locally. Moreover, unlike $[11,12]$, the exponential term was used in the construct of the filled function, which may increase the computability when applied to numerical optimization. Instead, in this paper, we use an efficient filled function method to solve the corresponding optimization problem, and the local minimizer of the filled function is always obtained in the interior and is always good point. The objective function value can be reduced by quarter in each iteration of our algorithm.

The existence of local minimizers other than global ones makes global optimization a great challenge. As one of the main methods to solve general unconstrained or boxconstrained global optimization problems without special structural property, the filled function method has attracted extensive attention; see [8-15]. The main idea of the filled function method is to construct an auxiliary function called filled function via the current local minimizer of the original optimization problem, with the property that the current local minimizer is a local maximizer of the constructed filled function and a better initial point of the primal optimization problem can be obtained by minimizing the constructed filled function locally. However, generally speaking, the local minimizer of the filled function cannot ensure that it is a better point of the primal optimization problem. In the paper, we propose a new filled function method, which can ensure that the proposed function is an efficient filled function and the local minimizer of the new filled function on a given box set is a better point and the primal problem's objective value at this better point can be reduced by quarter in each iteration of our filled function algorithm.

The numerical results obtained show that our method is applicable and efficient. The paper is organized as follows. Following this introduction, a novel filled function is proposed for the optimization problem in Section 2. The corresponding algorithm is presented in Section 3. In Section 4, several numerical examples are reported. Finally, some conclusions are drawn in Section 5.

\section{Filled Function for the Optimization Problem}

Throughout this paper we make the following assumption.

Assumption 1. (SNE) has at least one solution in $X$ and the number of solutions of (SNE) is finite.

Suppose that $x^{*}$ is a local miminizer of problem (OP), the definition of the filled function is as follows.

Definition 2. A continuously differentiable function $p\left(x, x^{*}\right)$ is called a filled function of problem (OP) at $x^{*}$, if it satisfies the following conditions:

(1) $x^{*}$ is a strict local maximizer of $p\left(x, x^{*}\right)$ on $X$;

(2) $p\left(x, x^{*}\right)$ has no stationary point in the region $S_{1}=$ $\left\{x: f(x)>f\left(x^{*}\right) / 4, x \in X \backslash\left\{x^{*}\right\}\right\}$
(3) If $x^{*}$ is not a global minimizer of problem (OP), then $p\left(x, x^{*}\right)$ does have a minimizer in the region $S_{2}=\{x:$ $\left.f(x) \leq f\left(x^{*}\right) / 4, x \in X\right\}$.

These conditions of the new filled function ensure that when a descent method, for example, the steepest descent method, is employed to minimize the constructed filled function, the sequence of iteration points will not terminate at any point at which the objective function value is large than $f\left(x^{*}\right) / 4$; if $x^{*}$ is not a global minimizer of problem (OP), then there must exist a minimizer of the filled function at which the objective function value is less than or equal to $f\left(x^{*}\right) / 4$, namely, any local minimizer of $p\left(x, x^{*}\right)$ must belong to the set $S_{2}=\left\{x: f(x) \leq f\left(x^{*}\right) / 4, x \in X\right\}$. Therefore, the present local minimizer of the objective function escapes and a better minimizer can be found by a local search algorithm starting from the minimizer of the filled function.

Let $L(P)$ denote the set of local minimizers of problem $(\mathrm{OP})$ and let $G(P)$ denote the set of global minimizers of problem (OP).

In the following, a novel filled function with one parameter satisfying Definition 2 is introduced. To begin with, we design a continuously differentiable function $h(t)$ with the following properties: it is equal to 0 when $t>0$.

More specifically, we construct $h(t)$ as follows:

$$
h(t)= \begin{cases}0, & t>0 \\ t^{2}, & t \leq 0\end{cases}
$$

It is not difficult to check that $h(t)$ is continuously differentiable and decreasing on $R$. Obviously, we have

$$
h^{\prime}(t)= \begin{cases}0, & t>0 \\ 2 t, & t \leq 0\end{cases}
$$

Given $x^{*} \in L(P)$, the following filled function with one parameter is constructed:

$$
F\left(x, x^{*}, q\right)=\frac{1}{1+\left\|x-x^{*}\right\|}+q h\left(f(x)-\frac{f\left(x^{*}\right)}{4}\right),
$$

where the only parameter $q>0$. Clearly, $F\left(x, x^{*}, q\right)$ is continuously differentiable on $R^{n}$.

The following theorems show that $F\left(x, x^{*}, q\right)$ satisfy Definition 2 when the positive parameter $q$ is sufficiently large.

Theorem 3. Let $x^{*} \in L(P), q>0$. Then, $x^{*}$ is a strict local maximizer of $F\left(x, x^{*}, q\right)$ on $X$.

Proof. Since $x^{*} \in L(P)$, there exists a neighborhood $N\left(x^{*}, \sigma\right)$ of $x^{*}$ with $\sigma>0$ such that $f(x) \geq f\left(x^{*}\right)$, for all $x \in N\left(x^{*}, \sigma\right)$, where $N\left(x^{*}, \sigma\right)=\left\{x \mid\left\|x-x^{*}\right\|<\sigma\right\}$. Then, for any $x \in$ $N\left(x^{*}, \sigma\right), x \neq x^{*}, q>0$, and $h(t) \geq 0$. We have

$$
F\left(x, x^{*}, q\right)=\frac{1}{1+\left\|x-x^{*}\right\|}<1=F\left(x^{*}, x^{*}, q\right) .
$$

Thus, $x^{*}$ is a strict local maximizer of $F\left(x, x^{*}, q\right)$ on $X$. 
Theorem 3 reveals that the proposed new filled function satisfies condition (1) of Definition 2.

Theorem 4. Let $x^{*} \in L(P), q>0$. Then, $F\left(x, x^{*}, q\right)$ has no stationary point in the region $S_{1}=\left\{x: f(x)>f\left(x^{*}\right) / 4, x \in\right.$ $\left.X \backslash\left\{x^{*}\right\}\right\}$.

Proof. Assume that $\bar{x} \in S_{1}$, namely, $f(\bar{x})>f\left(x^{*}\right) / 4$ and $\bar{x} \neq x^{*}$.

Then, we have

$$
\begin{gathered}
\nabla F\left(\bar{x}, x^{*}, q\right)=\frac{-\left(\bar{x}-x^{*}\right)}{\left(1+\left\|\bar{x}-x^{*}\right\|\right)^{2}\left\|\bar{x}-x^{*}\right\|}, \\
\nabla F\left(\bar{x}, x^{*}, q\right) \frac{\left(\bar{x}-x^{*}\right)}{\left\|\bar{x}-x^{*}\right\|}=\frac{-1}{\left(1+\left\|\bar{x}-x^{*}\right\|\right)^{2}}<0 .
\end{gathered}
$$

It implies that the function $F\left(x, x^{*}, q\right)$ has no stationary point in the region $S_{1}=\left\{x: f(x)>f\left(x^{*}\right) / 4, x \in X \backslash\left\{x^{*}\right\}\right\}$.

Theorem 4 reveals that the proposed new filled function satisfies condition (2) of Definition 2.

Theorem 5. Let $x^{*} \in L(P)$, but $x^{*} \notin G(P)$. And (SNE) satisfies Assumption 1. Then, $F\left(x, x^{*}, q\right)$ does have a minimizer in the region $S_{2}=\left\{x: f(x) \leq f\left(x^{*}\right) / 4, x \in X\right\}$ when $q>0$ is sufficiently large.

Proof. Since $x^{*} \in L(P)$, but $x^{*} \notin G(P)$, and the global minimum of $f(x)$ is zero, there exists an $\bar{x}^{*} \in L(\mathrm{OP})$ such that $f\left(\bar{x}^{*}\right) \leq f\left(x^{*}\right) / 4$. By the continuity of $f(x)$ and Assumption 1, there exists $\sigma>0$ that is small enough and $\bar{x} \in N\left(\bar{x}^{*}, \sigma\right)$, and it holds $f\left(\bar{x}^{*}\right) \leq f(x) \leq f\left(x^{*}\right) / 4=f(\bar{x})$, for all $x \in N\left(\bar{x}^{*}, \sigma\right)$, where $N\left(\bar{x}^{*}, \sigma\right)=\left\{x \in X:\left\|x-\bar{x}^{*}\right\|<\sigma\right\}$.

Here, we just give the proof to the case when $\left\|\bar{x}-x^{*}\right\| \leq$ $\left\|\bar{x}^{*}-x^{*}\right\|$. For the other case when $\left\|\bar{x}-x^{*}\right\|>\left\|\bar{x}^{*}-x^{*}\right\|$, the proof is similar.

Therefore, for each $x \in N(\bar{x}, \sigma)=\{x \in X:\|x-\bar{x}\|<\sigma\}$, there are two cases:

(1) $f(x)>f(\bar{x})=f\left(x^{*}\right) / 4$;

(2) $f(x) \leq f(\bar{x})=f\left(x^{*}\right) / 4$.

For case (1), by $f(x)-f\left(x^{*}\right) / 4>f(\bar{x})-f\left(x^{*}\right) / 4=0$ and $\left\|x-x^{*}\right\|<\left\|\bar{x}-x^{*}\right\|$

$$
F\left(x, x^{*}, q\right)=\frac{1}{1+\left\|x-x^{*}\right\|}>\frac{1}{1+\left\|\bar{x}-x^{*}\right\|}=F\left(\bar{x}, x^{*}, q\right) \text {. }
$$

For case (2), by $f(x)-f\left(x^{*}\right) / 4 \leq f(\bar{x})-f\left(x^{*}\right) / 4=0$ and $\left\|\bar{x}-x^{*}\right\|<\left\|x-x^{*}\right\|$, we have $F\left(\bar{x}, x^{*}, q\right)<F\left(x, x^{*}, q\right)$ if and only if

$$
\frac{1}{1+\left\|\bar{x}-x^{*}\right\|}<\frac{1}{1+\left\|x-x^{*}\right\|}+q\left(f(x)-\frac{f\left(x^{*}\right)}{4}\right)^{2},
$$

which is equivalent to

$$
q>\frac{\left\|x-x^{*}\right\|-\left\|\bar{x}-x^{*}\right\|}{\left(f(x)-f\left(x^{*}\right) / 4\right)^{2}\left(1+\left\|\bar{x}-x^{*}\right\|\right)\left(1+\left\|x-x^{*}\right\|\right)}>0 .
$$

Let

$$
q_{0}=\frac{\left\|x-x^{*}\right\|-\left\|\bar{x}-x^{*}\right\|}{\left(f(x)-f\left(x^{*}\right) / 4\right)^{2}\left(1+\left\|\bar{x}-x^{*}\right\|\right)\left(1+\left\|x-x^{*}\right\|\right)} .
$$

Thus, there exists sufficiently large $q_{0}>0$ as function $f(x)$ approaches $f\left(x^{*}\right) / 4$. Consequently, it must has that $F\left(\bar{x}, x^{*}, q\right)<F\left(x, x^{*}, q\right)$ for all $x \in N(\bar{x}, \sigma)$ when $q>q_{0}$. Thus, $\bar{x} \in S_{2}$ is a minimizer of $F\left(x, x^{*}, q\right)$ when $q>0$ is sufficiently large.

Theorems 5 show that, for all $q \geq q_{0}, F\left(x, x^{*}, q\right)$ satisfies Condition (3) of Definition 2. The following theorems show that function $F\left(x, x^{*}, q\right)$ has some interesting properties.

Theorem 6. Let $x_{1}, x_{2} \in X$ and the following conditions hold:

(i) $\min \left\{f\left(x_{1}\right), f\left(x_{2}\right)\right\} \geq(1 / 4) f\left(x^{*}\right)$;

(ii) $\left\|x_{2}-x^{*}\right\|>\left\|x_{1}-x^{*}\right\|$.

Then, the inequality $F\left(x_{1}, x^{*}, q\right)>F\left(x_{2}, x^{*}, q\right)$ holds for all $q>0$.

Proof. Since $\min \left\{f\left(x_{1}\right), f\left(x_{2}\right)\right\} \geq(1 / 4) f\left(x^{*}\right)$, then

$$
\begin{aligned}
& F\left(x_{1}, x^{*}, q\right)=\frac{1}{1+\left\|x_{1}-x^{*}\right\|}, \\
& F\left(x_{2}, x^{*}, q\right)=\frac{1}{1+\left\|x_{2}-x^{*}\right\|} .
\end{aligned}
$$

Therefore, for all $q>0, F\left(x_{1}, x^{*}, q\right)>F\left(x_{2}, x^{*}, q\right)$ holds.

Theorem 7. $F\left(x, x^{*}, q\right)>0$ for all $x \in X$.

Proof. By the form of the filled function (3) and since $h(t) \geq 0$, we have $F\left(x, x^{*}, q\right)>0$ for all $x \in X$.

Remark 8. In the phase of minimizing the filled function, Theorems 3-5 guarantee that the present local minimizer $x^{*}$ of the objective function is escaped and the minimum of the filled function will be always achieved at a point where the objective function value is not greater than the quarter of the current minimum of the objective function. Moreover, the proposed filled function does not include exponential terms. A continuously differentiable function is used in the constructed filled function, which possesses many good properties and is efficient in numerical implementation.

\section{Filled Function Algorithm}

The theoretical properties of the proposed filled function $F\left(x, x^{*}, q\right)$ were discussed in the last section. In this section, a global optimization method for solving problem (OP) is presented based on the constructed filled function (3), which leads to a solution or an approximate solution to (SNE).

Suppose that (SNE) has at least one solution and the number of solutions is finite. The general idea of the global optimization method is as follows. 
Let $x_{0} \in X$ be a given initial point. Starting from this initial point, a local minimizer $x_{0}^{*}$ of problem (OP) is obtained with a local minimization method (Newton method, QuasiNewton Method, or Conjugate Gradient method). If $x_{0}^{*}$ is not a global minimizer, the main task is to find a better local minimizer of problem (OP).

Consider the following filled function problem (for short $(\mathrm{FFP}))$ :

$$
\min _{x \in X} F\left(x, x_{k}^{*}, q\right),
$$

where $F\left(x, x_{k}^{*}, q\right)$ is given by $(3)$.

Let $\bar{x}_{0}^{*}$ be a obtained local minimizer of problem (FFP) on $X$, and then by Theorem 5 , we have $f\left(\bar{x}_{0}^{*}\right) \leq f\left(x_{0}^{*}\right) / 4$. Starting from this initial point $\bar{x}_{0}^{*}$, we can obtain a local minimizer $x_{1}^{*}$ of problem (OP). If $x_{1}^{*}$ is a global minimizer (namely, $f\left(x_{1}^{*}\right)=$ $0), x_{1}^{*}$ is the solution of the system (SNE); otherwise, locally solve problem (FFP). Let $\bar{x}_{1}^{*}$ be the obtained local minimizer, and then we have that $f\left(\bar{x}_{1}^{*}\right) \leq f\left(x_{0}^{*}\right) / 4^{2}$. Repeating this process, we can finally obtain a solution of the system (SNE) or a sequence $\left\{\bar{x}_{k}^{*}\right\}$ with $f\left(\bar{x}_{k}^{*}\right)<f\left(x_{0}^{*}\right) / 4^{k}, k=1,2, \ldots$. For such a sequence $\left\{\bar{x}_{k}^{*}\right\}, k=1,2, \ldots$, when $k$ is sufficiently large, $\bar{x}_{k}^{*}$ can be regarded as an approximate solution of the system (SNE).

Let $x^{*} \in X$ and $\epsilon>0$, and $x^{*}$ is called a $\epsilon$-approximate solution of the system (SNE) if $x^{*} \in X$ and $f\left(x^{*}\right) \leq \epsilon$.

The corresponding filled function algorithm for the global optimization problem (OP) is described as follows. The algorithm is referred as FFSNE (the filled function method for (SNE)).

\section{Algorithm FFSNE}

Step 0. Choose small positive numbers $\epsilon, \lambda$, a large positive number $q^{U}$, and an initial value $q_{0}$ for the parameters $q$. (e.g., $\epsilon=10^{-8}, \lambda=10^{-5}, q^{U}=10^{20}$, and $\left.q_{0}=10^{10}\right)$. Choose a positive integer number $K$ (e.g., $K=2 n$ ) and directions $e_{i}$, $i=1, \ldots, K$, are the coordinate directions. Choose an initial point $x_{0} \in X$. Set $k:=0$.

If $f\left(x_{0}\right) \leq \epsilon$, then let $x_{k}^{*}:=x_{0}$ and go to Step 6. Otherwise, let $q:=q_{0}$ and go to Step 1 .

Step 1. Find a local minimizer $x_{k}^{*}$ of the problem (OP) by local search methods starting from $x_{k}$. If $f\left(x_{k}^{*}\right) \leq \epsilon$, go to Step 6 .

Step 2. Let

$$
F\left(x, x_{k}^{*}, q\right)=\frac{1}{1+\left\|x-x_{k}^{*}\right\|}+q h\left(f(x)-\frac{f\left(x_{k}^{*}\right)}{4}\right),
$$

where $h(t)$ is defined by (1). Set $l=1$ and $u=0.1$.

\section{Step 3. Consider}

(a) If $l>K$, set $q:=10 q$, and go to Step 5; otherwise, go to $(\mathrm{b})$.

(b) If $u \geq \lambda$, set $y_{k}^{l}:=x_{k}^{*}+u e_{l}$, and go to (c); otherwise, set $l:=l+1, u=0.1$, go to (a).

(c) If $y_{k}^{l} \in X$, go to (d); otherwise, set $u:=u / 10$, go to (b). (d) If $f\left(y_{k}^{l}\right) \leq f\left(x_{k}^{*}\right) / 4$, then set $x_{k+1}:=y_{k}^{l}, k:=k+1$, and go to Step 1; otherwise, go to Step 4 .

Step 4. Search for a local minimizer of the following filled function problem starting from $y_{k}^{l}$ :

$$
\min _{x \in R^{n}} F\left(x, x_{k}^{*}, q\right) .
$$

Once a point $y_{k}^{*} \in X$ with $f\left(y_{k}^{*}\right) \leq f\left(x_{k}^{*}\right) / 4$ is obtained in the process of searching, set $x_{k+1}:=y_{k}^{*}, k:=k+1$ and go to Step 1; otherwise continue the process. Let $\bar{x}_{k}^{*}$ be an obtained local minimizer of problem (12). If $\bar{x}_{k}^{*}$ satisfies $f\left(\bar{x}_{k}^{*}\right) \leq f\left(x_{k}^{*}\right) / 4$, then set $x_{k+1}:=\bar{x}_{k}^{*}, k:=k+1$ and go to Step 1; otherwise, set $u:=u / 10$, and go to Step 3(b).

Step 5. If $q \leq q^{U}$, go to Step 2 .

Step 6. Let $x_{s}=x_{k}^{*}$ and stop.

In this algorithm, the termination criteria for minimization of $F\left(x, x_{k}^{*}, q\right)$ in Step 4 can be interpreted as follows. The purpose of minimizing $F\left(x, x_{k}^{*}, q\right)$ is to find a "better" point $y_{k}^{*}$ in set $S_{2}=\left\{x: f(x) \leq f\left(x^{*}\right) / 4, x \in X\right\}$. If it is successful, that is, the solution obtained satisfies $y_{k}^{*} \in S_{2}$, then we can turn to Step 1 and restart to minimize the objective function with $y_{k}^{*}$ as a new starting point. If in the process of searching such point is not found, we can obtain the local minimizer $\bar{x}_{k}^{*}$ of $F\left(x, x_{k}^{*}, q\right)$. By Theorems 4 and 5 , we know that there must exist a local minimizer of $F\left(x, x_{k}^{*}, q\right)$ which belongs to the set $S_{2}=\left\{x: f(x) \leq f\left(x^{*}\right) / 4, x \in X\right\}$. Then, we turn to Step 1 and minimizer $f(x)$ starting from the local minimizer $\bar{x}_{k}^{*}$. Obviously, if $\lambda$ is small enough, $q^{U}$ is large enough, and the direction set $\left\{e_{1}, \ldots, e_{K}\right\}$ is large enough and $x_{s}$ can be obtained from algorithm FFOP within finite steps.

\section{Numerical Experiment}

In this section, several sets of numerical experiments are presented to illustrate the efficiency of algorithm FFSNE. All the numerical experiments are implemented in Matlab2010b. In our programs, the local minimizers of problem (FFP) and problem (OP) are obtained by the SQP method. Note that $\|\nabla f(x)\| \leq 10^{-6}$ is used as the terminate condition.

The symbols used in Table 3 are given in Table 1 .

Throughout our computational experiments, the parameters in algorithm FFSNE are set as

$$
\varepsilon=10^{-8}, \quad \lambda=10^{-5}, \quad q^{U}:=10^{20}, \quad q_{0}:=10^{10} .
$$

Problem 9 (test problem 14.1.1 in [16]). Consider

$$
\begin{gathered}
4 x_{1}^{3}+4 x_{1} x_{2}+2 x_{2}^{2}-42 x_{1}-14=0 \\
4 x_{2}^{3}+2 x_{1}^{2}+4 x_{1} x_{2}-26 x_{2}-22=0 \\
-5 \leq x_{i} \leq 5, \quad i=1,2 .
\end{gathered}
$$

There are nine known solutions as shown in [16] (see Table 2). 
TABLE 1

\begin{tabular}{ll}
\hline Parameter & Description \\
\hline$k$ & $\begin{array}{l}\text { The number of iterations in finding the } k \text { th local } \\
\text { minimizer }\end{array}$ \\
$x_{0}$ & $\begin{array}{l}\text { The initial point which satisfies } x_{0} \in X \\
x_{k}^{*}\end{array}$ \\
$\begin{array}{l}\text { The } k \text { th local minimizer which we take as a } \\
\text { solution or an approximate solution to (SNE) }\end{array}$ \\
$\begin{array}{l}\text { The function value of } f(x) \text { in finding the } k \text { th local } \\
\text { minimizer }\end{array}$ \\
$F\left(x_{k}^{*}\right)$ & $\begin{array}{l}\text { The function value of } F(x) \text { in finding the } k \text { th local } \\
\text { minimizer }\end{array}$ \\
\hline
\end{tabular}

Problem 10 (test problem 14.1.2 in [16]). Consider

$$
\begin{gathered}
x_{1} x_{2}+x_{1}-3 x_{5}=0, \\
2 x_{1} x_{2}+x_{1}+3 R_{10} x_{2}^{2}+x_{2} x_{3}^{2}+R_{7} x_{2} x_{3} \\
+R_{9} x_{2} x_{4}+R_{8} x_{2}-R x_{5}=0, \\
2 x_{2} x_{3}^{2}+R_{7} x_{2} x_{3}+2 R_{5} x_{3}^{2}+R_{6} x_{3}-8 x_{5}=0, \\
R_{9} x_{2} x_{4}+2 x_{4}^{2}-4 R x_{5}=0, \\
x_{1} x_{2}+x_{1}+R_{10} x_{2}^{2}+x_{2} x_{3}^{2}+R_{7} x_{2} x_{3}+R_{9} x_{2} x_{4} \\
+R_{8} x_{2}+R_{5} x_{3}^{2}+R_{6} x_{3}+x_{4}^{2}-1=0, \\
0.0001 \leq x_{i} \leq 100, \quad i=1, \ldots, 5,
\end{gathered}
$$

where $R=10, R_{5}=0.193, R_{6}=4.10622 \times 10^{-4}, R_{7}=$ $5.45177 \times 10^{-4}, R_{8}=4.4975 \times 10^{-7}, R_{9}=3.40735 \times 10^{-5}$, and $R_{10}=9.615 \times 10^{-7}$.

The known solution in [16] is $(0.003431,31.325636,0.068352,0.859530,0.036963)^{T}$.

Problem 11 (test problem 14.1.3 in [16]). Consider

$$
\begin{gathered}
10^{4} x_{1} x_{2}-1=0, \\
\exp \left(-x_{1}\right)+\exp \left(-x_{2}\right)-1.001=0, \\
5.49 \times 10^{-6} \leq x_{1} \leq 4.553 \\
2.196 \times 10^{-3} \leq x_{2} \leq 18.21
\end{gathered}
$$

The known solution is $(0.0000145067,6.89335287)^{T}$.

Problem 12 (test problem 14.1.4 in [16]). Consider

$$
\begin{gathered}
0.5 \sin \left(x_{1} x_{2}\right)-\frac{0.25 x_{2}}{\pi}-0.5 x_{1}=0, \\
\left(1-\frac{0.25}{\pi}\right)\left(e^{2 x_{1}}-e\right)+\frac{e x_{2}}{\pi}-2 e x_{1}=0, \\
0.25 \leq x_{1} \leq 1, \quad 1.5 \leq x_{2} \leq 2 \pi .
\end{gathered}
$$

The known solution is $(0.29945,2.83693)^{T}$ and $(0.5,3.14159)^{T}$.
Problem 13 (test problem 14.1.5 in [16]). Consider

$$
\begin{gathered}
2 x_{1}+x_{2}+x_{3}+x_{4}+x_{5}-6=0, \\
x_{1}+2 x_{2}+x_{3}+x_{4}+x_{5}-6=0, \\
x_{1}+x_{2}+2 x_{3}+x_{4}+x_{5}-6=0, \\
x_{1}+x_{2}+x_{3}+2 x_{4}+x_{5}-6=0, \\
x_{1} x_{2} x_{3} x_{4} x_{5}-1=0 \\
-2 \leq x_{i} \leq 2, \quad i=1, \ldots, 5 .
\end{gathered}
$$

The known solution is $(1,1,1,1,1)^{T}$ and $(0.916,0.916,0.916,0.916,1.418)^{T}$.

Problem 14 (test problem 14.1.6 in [16]). Consider

$$
\begin{gathered}
4.731 \times 10^{-3} x_{1} x_{3}-0.3578 x_{2} x_{3}-0.1238 x_{1} \\
+x_{7}-1.637 \times 10^{-3} x_{2}-0.9338 x_{4}-0.3571=0, \\
0.2238 x_{1} x_{3}+0.7623 x_{2} x_{3}+0.2638 x_{1}-x_{7} \\
-0.07745 x_{2}-0.6734 x_{4}-0.6022=0 \\
x_{6} x_{8}+0.3578 x_{1}+4.731 \times 10^{-3} x_{2}=0 \\
-0.7623 x_{1}+0.2238 x_{2}+0.3461=0 \\
x_{1}^{2}+x_{2}^{2}-1=0 \\
x_{3}^{2}+x_{4}^{2}-1=0 \\
x_{5}^{2}+x_{6}^{2}-1=0 \\
x_{7}^{2}+x_{8}^{2}-1=0 \\
-1 \leq x_{i} \leq 1, \quad i=1, \ldots, 8
\end{gathered}
$$

The are sixteen known solutions of problem 6 are given in [16].

Problem 15 (test problem 1 in [17]). Consider

$$
\begin{gathered}
1-2 x_{2}+0.2 \sin \left(4 \pi x_{2}\right)-x_{1}=0, \\
x_{2}-0.5 \sin \left(2 \pi x_{1}\right)=0, \\
-10 \leq x_{1}, \quad x_{2} \leq 10 .
\end{gathered}
$$

The known solution is $(0.1025250,0.3005036)^{T}$.

Problem 16 (test problem 7 in [18]). Consider

$$
\begin{gathered}
x_{1}-0.7 \sin \left(x_{1}\right)-0.2 \cos \left(x_{2}\right)=0, \\
x_{2}-0.7 \cos \left(x_{1}\right)+0.2 \sin \left(x_{2}\right)=0, \\
-100 \leq x_{1}, \quad x_{2} \leq 100 .
\end{gathered}
$$

The known solution is $(0.5268,0.5084)^{T}$. 
TABLE 2

\begin{tabular}{lccccccccc}
\hline Sol & 1 & 2 & 3 & 4 & 5 & 6 & 7 & 8 & 9 \\
$x_{1}^{*}$ & -3.7793 & -3.0730 & -2.8051 & -0.2709 & -0.1280 & 0.0867 & 3.0 & 3.3852 & 3.5844 \\
$x_{2}^{*}$ & -3.2832 & -0.0814 & 3.1313 & -0.9230 & -1.9537 & 2.8843 & 2.0 & 0.0739 & -1.8481 \\
\hline
\end{tabular}

TABle 3: Computational results for Problems 9-16.

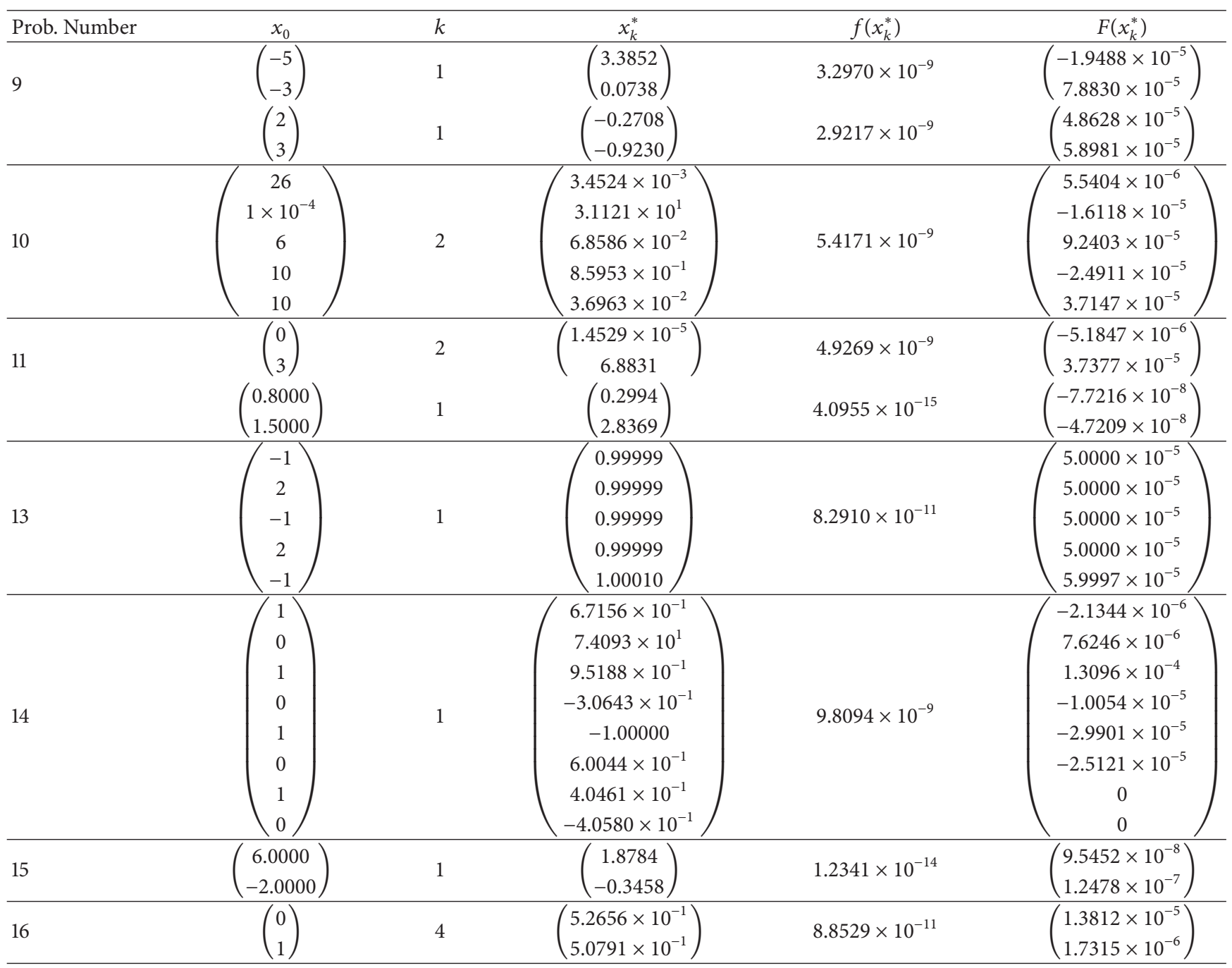

The numerical results are listed in Table 3. From Table 3, it is easy to see that all problems that we tested have been solved with a small number of iterations.

\section{Conclusions}

In this paper, the filled function $F\left(x, x^{*}, q\right)$ with one parameter is constructed for solving nonlinear equations and it has been proved that it satisfies the basic characteristics of the filled function definition. Promising computation results have been observed from our numerical experiments. In the future, the filled function method can be used to solve other problems such as nonlinear systems of equalities and inequalities and nonlinear feasibility problems with expensive functions.

\section{Conflict of Interests}

The authors declare that there is no conflict of interests regarding the publication of this paper.

\section{Acknowledgment}

This work was supported by the Natural Science Foundation of China (no. 71171150 and no. 51275366).

\section{References}

[1] S. Fucik and A. Kufner, Nonlinear Differential Equations, Scientific Publishing, New York, NY, USA, 2008. 
[2] X. Chen, L. Qi, and Y. F. Yang, "Lagrangian globalization methods for nonlinear complementarity problems," Journal of Optimization Theory and Applications, vol. 112, no. 1, pp. 77-95, 2002.

[3] C. Kanzow, "Global optimization techniques for mixed complementarity problems," Journal of Global Optimization, vol. 16, no. 1, pp. 1-21, 2000.

[4] J. L. Nazareth and L. Qi, "Globalization of Newton's methods for solving nonlinear equations," Numerical Linear Algebra with Applications, vol. 3, pp. 239-249, 1996.

[5] L. Qi and Y. F. Yang, "NCP functions applied to Lagrangian globalization for the nonlinear complementarity problem," Journal of Global Optimization, vol. 24, no. 2, pp. 261-283, 2002.

[6] X. J. Tong, L. Qi, and Y. F. Yang, "The Lagrangian globalization method for nonsmooth constrained equations," Computational Optimization and Applications, vol. 33, no. 1, pp. 89-109, 2006.

[7] A. V. Levy and A. Montalvo, "The tunneling algorithm for the global minimization of functions," SIAM Journal on Scientific and Statistical Computing, vol. 6, no. 1, pp. 15-29, 1985.

[8] R. P. Ge, "A filled function method for finding a global minimizer of a function of several variables," Mathematical Programming, vol. 46, no. 2, pp. 191-204, 1990.

[9] Z. Y. Wu, M. Mammadov, F. S. Bai, and Y. J. Yang, "A filled function method for nonlinear equations," Applied Mathematics and Computation, vol. 189, no. 2, pp. 1196-1204, 2007.

[10] Y. Lin, Y. J. Yang, and M. Mammadov, "A new filled function method for nonlinear equations," Applied Mathematics and Computation, vol. 210, no. 2, pp. 411-421, 2009.

[11] Y. Lin and Y. Yang, "Filled function method for nonlinear equations," Journal of Computational and Applied Mathematics, vol. 234, no. 3, pp. 695-702, 2010.

[12] C. J. Wang, R. H. Luo, K. Wu, and B. S. Han, "A new filled function method for an unconstrained nonlinear equation," Journal of Computational and Applied Mathematics, vol. 235, no. 6, pp. 1689-1699, 2011.

[13] Y. Lin and Y. Yang, "A filled function method for box constrained nonlinear integer programming," Journal of the Korean Mathematical Society, vol. 48, no. 5, pp. 985-999, 2011.

[14] L. Y. Yuan, Z. Wan, J. J. Zhang, and B. Sun, "A filled function method for solving nonlinear complementarity problem," Journal of Industrial and Management Optimization, vol. 5, no. 4, pp. 911-928, 2009.

[15] W. Zhu, "Globally concavized filled function method for the box constrained continuous global minimization problem," Optimization Methods and Software, vol. 21, no. 4, pp. 653-666, 2006.

[16] C. A. Floudas, P. M. Pardalos, C. S. Adjiman et al., Handbook of Test Problems in Local and Global Optimization, vol. 33, Kluwer Academic, Dordrecht, The Netherland, 1999.

[17] L. S. Zhang, C. K. Ng, D. Li, and W. W. Tian, "A new filled function method for global optimization," Journal of Global Optimization, vol. 28, no. 1, pp. 17-43, 2004.

[18] Y. Zhang and Z. H. Huang, "A nonmonotone smoothing-type algorithm for solving a system of equalities and inequalities," Journal of Computational and Applied Mathematics, vol. 233, no. 9, pp. 2312-2321, 2010. 


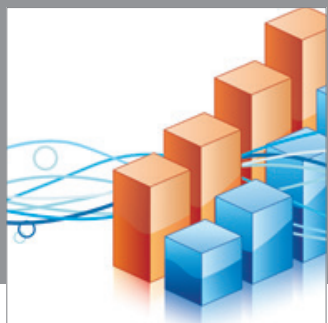

Advances in

Operations Research

mansans

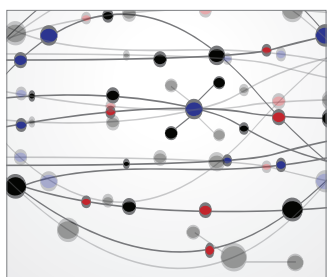

The Scientific World Journal
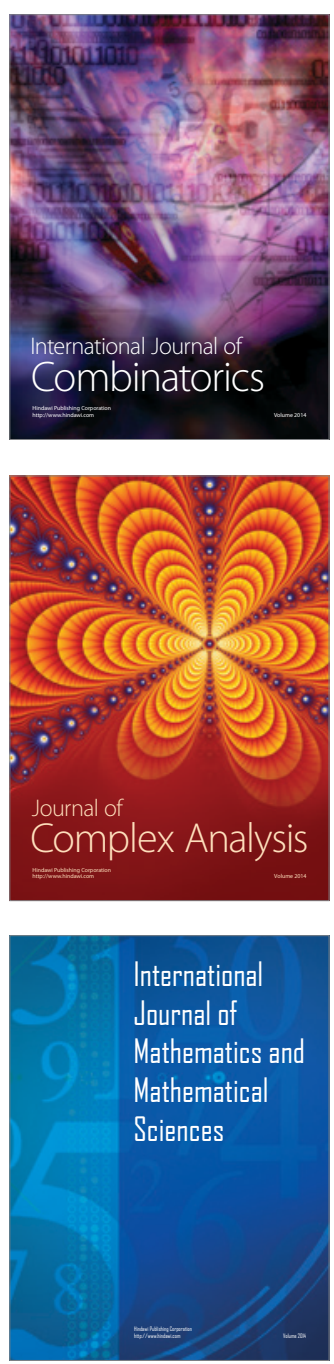
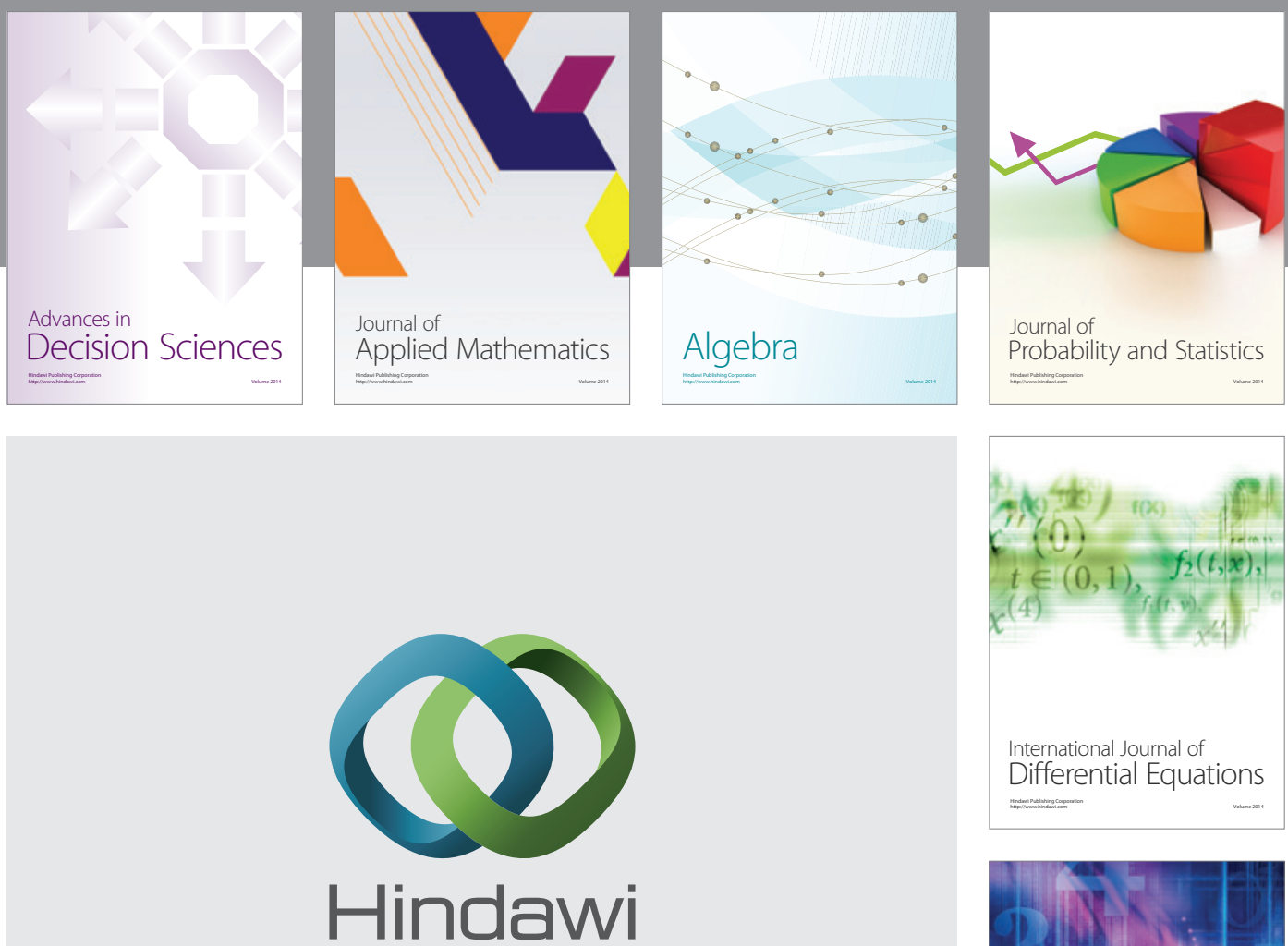

Submit your manuscripts at http://www.hindawi.com
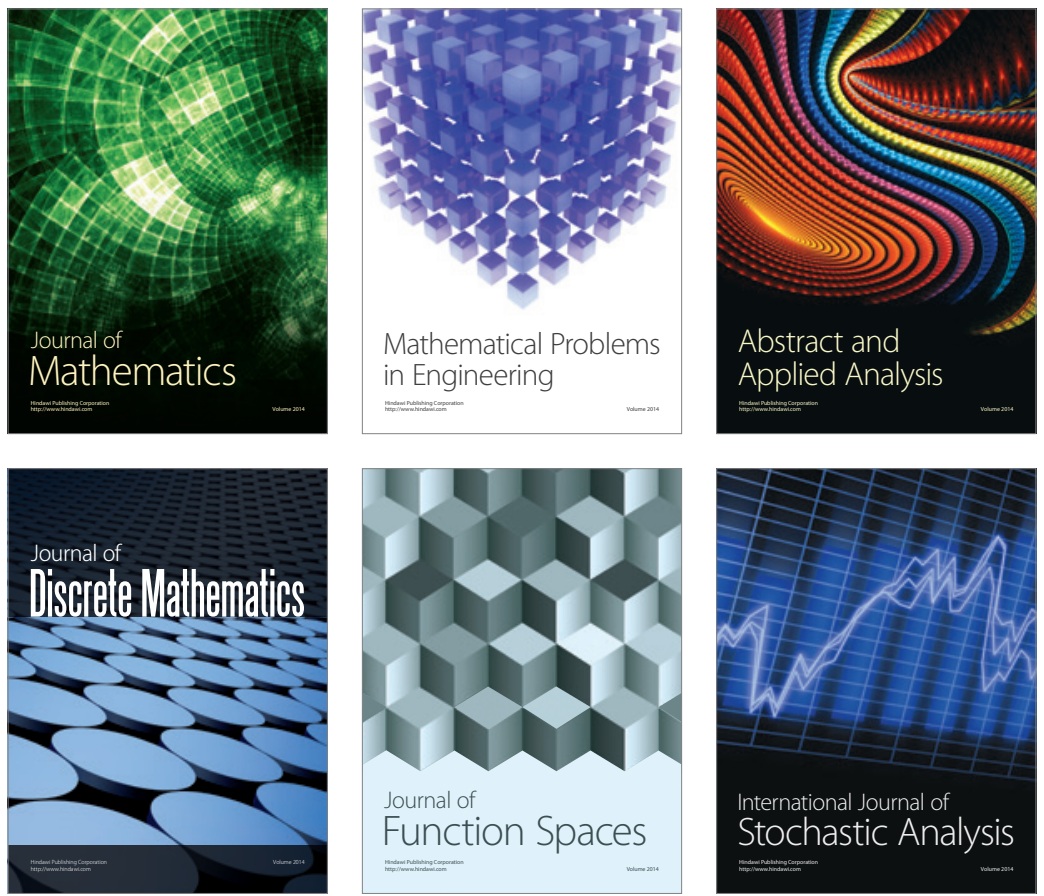

Journal of

Function Spaces

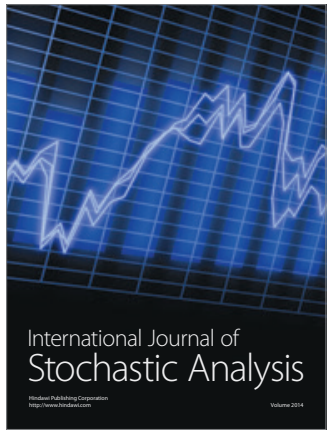

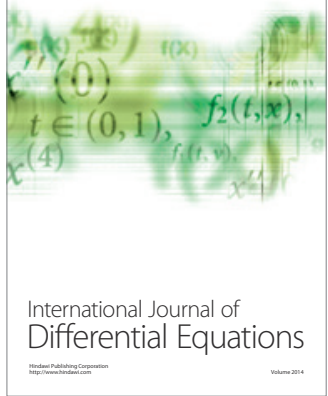
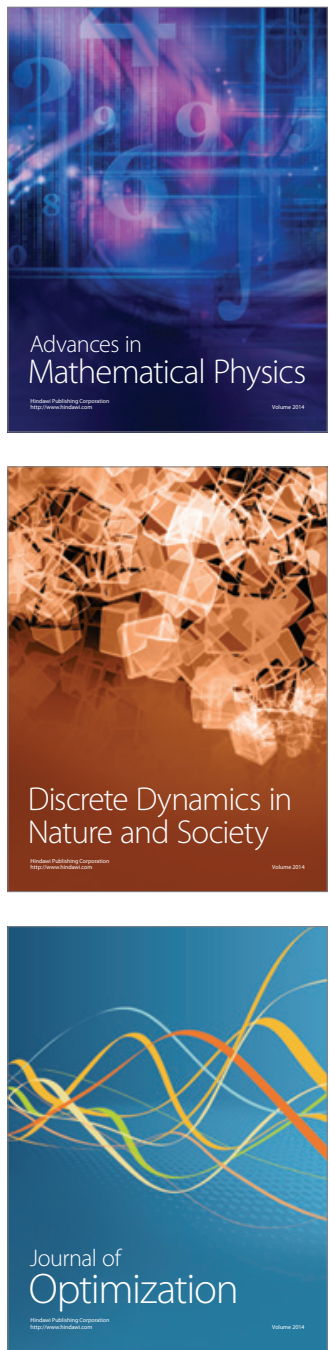